\title{
Software de fotoelasticidad RGB en placas de materiales birrefringentes al esfuerzo
}

\section{RGB photoelasticity software on plates of stress birefringent materials}

\author{
David Trejo Carrillo( ${ }^{(1)}$, Rubén Castañeda Balderas ${ }^{(1)}$, Alberto Díaz Díaz
}

Metalurgía e Integridad estructural, Centro de Investigación en Materiales Avanzados, S.C., Av. Miguel de Cervantes Saavedra 120, Complejo Industrial Chihuahua, 31136 Chihuahua, Chihuahua, México

Autor de correspondencia: David Trejo Carrillo, Centro de Investigación en Materiales Avanzados, S.C., Av. Miguel de Cervantes Saavedra 120, Complejo Industrial Chihuahua, 31136 Chihuahua, Chihuahua, México. E-mail: david.trejo@ cimav.edu.mx. ORCID: 0000-0001-8189-682X.

Recibido: 31 de Agosto del $2021 \quad$ Aceptado: 01 de Diciembre del $2021 \quad$ Publicado: 30 de Diciembre del 2021 Resumen.- La fotoelasticidad digital utiliza el fenómeno de la birrefringencia para el análisis y descripción de la distribución de esfuerzos a través de imágenes digitales y del procesamiento digital de las mismas. En este trabajo se presenta el desarrollo y diseño de un código, abierto y gratuito, en MATLAB que adopta la metodología de la fotoelasticidad RGB para determinar la distribución de la diferencia de esfuerzos principales en una probeta plana de un material fotoelástico, sometida a cargas mecánicas en un estado de esfuerzos planos. Esto mediante el procesamiento de una imagen digital a color de un espécimen de material birrefringente al esfuerzo, colocado dentro de un polariscopio circular en su configuración de campo oscuro. Se presenta la generación de imágenes artificiales representativas del fenómeno con el fin de tener datos de entrada para el desarrollo del algoritmo y una forma de validarlo. Se muestran también los pasos necesarios para la metodología de la fotoelasticidad RGB que incluyen la preparación de una base de datos o tabla de búsqueda y el análisis de la imagen fotoelástica. Se compara, además, el resultado del cálculo de la diferencia de esfuerzos principales con resultados de elemento finito, en lo cual se obtuvo un buen grado de confiabilidad en las imágenes utilizadas.

Palabras clave: Fotoelasticidad digital; Distribución de esfuerzos; Software; MATLAB.

\begin{abstract}
Digital photoelasticity uses the phenomenon of birefringence for the analysis and description of the stress distribution through digital images and their digital processing. This work presents the development and design of a free, open-source code in MATLAB that adopts the RGB photoelasticity methodology to determine the distribution of the principal stress difference in a flat specimen of a photoelastic material, subjected to mechanical loads in a state of flat stresses. This by processing a digital color image of a specimen of stress birefringent material, placed inside a circular polariscope in its dark field configuration. The generation of artificial images representative of the phenomenon is presented in order to have input data for the development of the algorithm and a way to validate it. The steps required for the RGB photoelasticity methodology are also shown, including preparing a database or lookup table and analyzing the photoelastic image. In addition, the result of the calculation of the difference in principal efforts is compared with the results of the finite element, in which a good degree of reliability was obtained in the images used.
\end{abstract}

Keywords: Digital photoelasticity; Stress distribution; Software; MATLAB. 


\section{Introducción}

La fotoelasticidad es una técnica que utiliza el fenómeno de la birrefringencia o doble refracción para el análisis y descripción de la distribución de esfuerzos [1]. Dicho fenómeno se debe a propiedades ópticamente anisótropas, presentes en ciertos materiales cristalinos; sin embargo, algunos materiales no cristalinos pueden exhibir birrefringencia cuando son sometidos a un esfuerzo [2], por ejemplo polímeros como el policarbonato, el poliuretano y el polimetilmetacrilato [3].

La técnica de la fotoelasticidad permite entonces, observar los esfuerzos a los que está sometido el material a través de patrones de interferencia producidos por el retardo causado en una onda electromagnética al pasar por el material birrefringente al esfuerzo [4, 5]. Para visualizar y analizar el fenómeno se requiere de un instrumento óptico conocido como polariscopio; se muestra un ejemplo en la Figura 1.

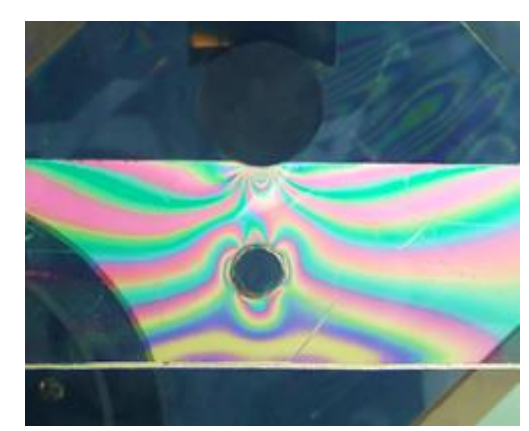

Figura 1. Ejemplo de un material birrefringente al esfuerzo visto a través del arreglo óptico del polariscopio.

Es pertinente señalar que la fotoelasticidad pertenece a las técnicas denominadas de campo completo [6]. Esto quiere decir que permite visualizar y determinar la distribución de esfuerzos a lo largo de toda la probeta sometida a carga [7]. Lo anterior representa una valiosa información en cuanto a la caracterización de materiales mediante pruebas mecánicas debido a que lo más habitual es obtener información del esfuerzo y la deformación en función del desplazamiento de la máquina que realiza la prueba o con la ayuda de sensores que ofrecen información puntual [8]. Por lo que el desarrollo e implementación de métodos fotoelásticos, como el desarrollo de un algoritmo de tipo libre y gratuito que implemente la fotoelasticidad digital, permitirá obtener información experimental invaluable para el avance de la ciencia de materiales. Sin embargo cabe destacar que la tendencia en cuanto al análisis de esfuerzos es utilizar dos o más métodos que proporcionen información cada vez más precisa $[1,9,10]$.

La fotoelasticidad ha tenido un papel considerable dentro de la historia de los métodos experimentales de análisis de esfuerzos. Se ha usado mucho antes del empleo de herramientas computacionales y tiene gran utilidad en analizar componentes de geometría compleja o con distribuciones de esfuerzos no uniformes debido, sobre todo, a que con un equipo relativamente sencillo es posible llegar a buenas aproximaciones [11]. Mediante el procesamiento digital de imágenes, se han hecho grandes avances en cuanto a la adquisición y procesamiento de los datos de manera automática. A esto se le conoce como fotoelasticidad digital que implica el uso de la técnica de la fotoelasticidad a través de imágenes digitales y el procesamiento digital de las mismas $[12,13,14]$.

Así pues, el desarrollo de técnicas de fotoelasticidad digital ha girado en torno a la búsqueda de una mayor automatización mediante el uso de las herramientas computacionales [14]. Para esto, han sido propuestas numerosas técnicas para la obtención de información fotoelástica precisa. Algunos de estos métodos han sido: adelgazamiento de franjas, desplazamiento de fase, desplazamiento de carga , tres longitudes de onda, análisis del transporte de franja, análisis espectral, escaneo de franjas, uso de la transformada de Fourier, y la fotoelasticidad RGB o también llamada de tres franjas $[15,16,17]$. 
En este trabajo se presenta una herramienta de software, abierta y gratuita, para describir la distribución de la diferencia de esfuerzos principales en probetas planas de un material fotoelástico sometido a cargas mecánicas; esto implementando la técnica de la fotoelasticidad digital RGB, mediante imágenes digitales, representativas del fenómeno, generadas artificialmente con la ayuda de los datos arrojados por una simulación de elemento finito de probetas de geometría sencilla.

\section{Metodología}

\subsection{Fotoelasticidad RGB}

De manera general, cualquier método que utilice el fenómeno de la fotoelasticidad pretende obtener información a partir del patrón formado y de la intensidad de la luz de las imágenes obtenidas a través del arreglo óptico. De forma más particular, el método de la fotoelasticidad RGB consiste en determinar el retardo relativo y con él, la distribución de la diferencia de esfuerzos principales, a través de una imagen, obtenida por una cámara digital, del espécimen de un material birrefringente al esfuerzo, en un polariscopio circular [17]. Dicho retardo está en función del campo de esfuerzo inducido, por lo que tendrá características distintas en cada punto de la pieza $[1,18]$; lo anterior por medio de la relación:

$$
\delta=\frac{2 \pi h}{\lambda} C\left(\sigma_{p 1}-\sigma_{p 2}\right)
$$

donde $h$ es el espesor del modelo, $\lambda$ la longitud de onda del rayo incidente, $\left(\sigma_{p 1}-\sigma_{p 2}\right)$ la diferencia de esfuerzos principales y $C$ es el coeficiente de esfuerzo óptico relacionado con el material. Es usual reescribir la ecuación 1 en términos del llamado orden de franja $(N)$ :

$$
N=\frac{\delta}{2 \pi}=\frac{h}{\lambda} C\left(\sigma_{p 1}-\sigma_{p 2}\right)
$$

y también de la forma conocida como ley de esfuerzo óptico:

$$
\left(\sigma_{p 1}-\sigma_{p 2}\right)=\frac{N \lambda}{h C}
$$

La fotoelasticidad RGB consiste básicamente en dos pasos: calibración y demodulación. En la calibración se crea una base de datos o tabla de búsqueda. La manera más común de generar la tabla de búsqueda $[19,20,21]$, es obtener los valores de los canales $R, G, B$ a lo largo de una línea en la sección transversal (ver Figura 2) de la imagen digital de una probeta plana, de material birrefringente, sometida a flexión pura $[17,14]$. Esto debido a que el esfuerzo varía de forma lineal a lo largo de la sección mencionada [22]. En este proyecto se propuso construir la tabla de búsqueda de manera teórica tomando como referencia el trabajo de Swain et al. [23].

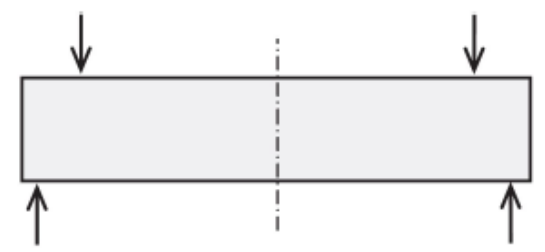

Figura 2. Ejemplo de una probeta a flexión. La línea punteada marca la sección de interés para generar la tabla de búsqueda.

\subsection{Tabla de búsqueda}

Por medio de un código en MATLAB, se elaboró la tabla de búsqueda según el siguiente procedimiento:

- Seleccionar el material del espécimen, así como determinar su altura y su espesor. Se eligió un modelo de $5 \mathrm{~mm}$ de espesor, $5 \mathrm{~cm}$ de altura y como material el polimetilmetacrilato que presenta un coeficiente fotoelástico (C) de $1.08 \times 10^{-10} \mathrm{~m}^{\wedge} 2 / N$ [7].

- Determinar el orden de franja máximo $\left(N_{\max }\right)$ y la resolución deseada. En este caso, resolución tiene que ver con la relación de franja por pixel. Se determinó el orden de franja 
máximo como 3 y una resolución de 0.01 franja/pixel.

- Calcular el valor del esfuerzo máximo en el punto más lejano del eje neutro de la probeta con la relación:

$$
\sigma_{\max }=\frac{N_{\max } \lambda_{G}}{h C}
$$

Es pertinente aclarar que se tomó como referencia la longitud de onda verde $\left(\lambda_{G}=\right.$ $545 \mathrm{~nm}$ [17]).

- Obtener el valor del esfuerzo en cualquier punto a lo largo de la línea seleccionada, a partir de

$$
\sigma_{i}=\sigma_{\max }\left(\frac{d_{i}}{d_{\max }}\right)
$$

donde $d$ es la distancia del eje neutro a la orilla del espécimen; en este caso sería $d=$ altura $/ 2$. - Con el resultado anterior, calcular en cada punto el valor del orden de franja para cada longitud de onda:

$$
\begin{aligned}
& N_{R}=\frac{h C \sigma_{i}}{\lambda_{R}} \\
& N_{G}=\frac{h C \sigma_{i}}{\lambda_{G}} \\
& N_{B}=\frac{h C \sigma_{i}}{\lambda_{B}}
\end{aligned}
$$

donde $N_{R}, N_{G}, N_{B}$, son los respectivos valores de orden de franja para cada una de las longitudes de onda utilizadas $\left(\lambda_{R}=610 \mathrm{~nm}\right.$ y $\lambda_{B}=$ $435 \mathrm{~nm}$ [24]).

- $\quad$ Finalmente, se calcula el valor del nivel de intensidad para cada uno de los canales R, G, B:

$$
I_{k}(i)=I_{k_{\max }} \operatorname{sen}^{2}\left(N_{k_{i}} \pi\right)
$$

que es la expresión clásica de la intensidad [1] de un polariscopio circular para una fuente de luz monocromática, donde $I_{k}$ es la intensidad para cada una de las longitudes de onda $(R, G, B)$, $I_{k_{\max }}$ la intensidad máxima de la fuente de luz y $k$ los canales $R, G, B$.

Con esta información se construye la tabla de búsqueda. En la Figura 3 se presenta la curva RGB, o el valor de la intensidad para cada color visto en un espacio tridimensional; esto presenta de manera intuitiva la línea a la que debe encontrarse la distancia más corta al relacionar la tabla de búsqueda con alguna imagen a analizar.

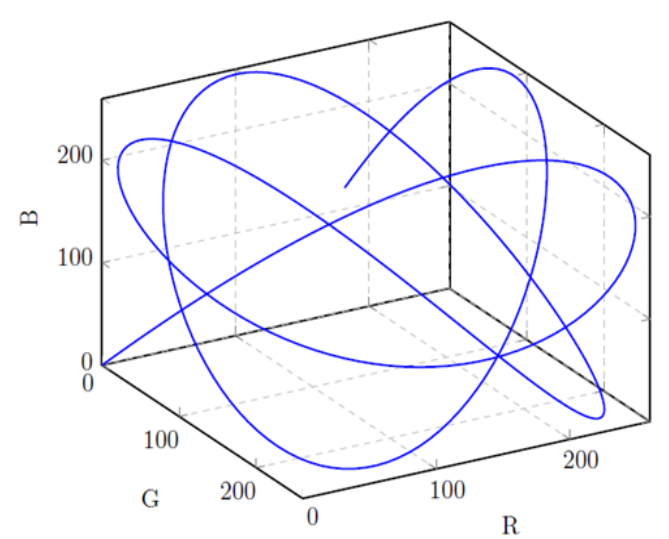

Figura 3. Curva RGB.

\subsubsection{Adaptación de la tabla de búsqueda}

Al elaborar la tabla de búsqueda, lo ideal es que las intensidades de los colores resultantes sean lo más parecidas a las de la imagen a analizar para realizar una mejor correlación. Sin embargo, debido a ciertas variaciones que pudieran resultar [25] es recomendable hacer una adaptación de color [14].

Se han propuesto algunas metodologías para subsanar dicha situación. Una de ellas es normalizar la imagen del espécimen a través de filtros digitales [26, 27], y otra manera es adaptar los datos de la tabla de búsqueda a las intensidades de la imagen que es lo que se adopta en este trabajo basado en Swain et al. [19] donde se sugiere un esquema de adaptación de color mediante una interpolación cuadrática de tres 


\section{Revista de Ciencias Tecnológicas (RECIT): Volumen 4 (4): 399-411}

puntos. De esta manera se obtiene una nueva tabla de búsqueda.

\subsection{Búsqueda del retardo}

Es conveniente recordar que una imagen digital puede considerarse como una matriz de valores de pixeles; las imágenes RGB o a color se representan como un arreglo numérico de $m \times n \times 3$ [28]. Es decir, que hay tres matrices distintas para los valores de intensidad de los canales rojo, verde y azul, respectivamente. El rango de los valores numéricos para cada pixel varía dependiendo del tipo de dato utilizado que puede ser de 0 a 1 (single o double) o de 0 a 255 (uint8) [29]. Dicho esto, el segundo paso de la fotoelasticidad RGB, llamado demodulación, consiste en obtener información de la imagen fotoelástica; para esto se requiere determinar el retardo en cualquier punto del área a analizar a través de sus niveles de intensidad para cada canal R, G, B mediante una comparación con los valores previamente guardados en la tabla de búsqueda. Hay que mencionar que por lo general la tabla se construye en términos del orden de franja (ecuación (2)), y este se obtiene al minimizar el valor de una función para cada pixel de la imagen fotoelástica [19]:

$$
e_{i}=\sqrt{\left(R-R_{i}\right)^{2}+\left(G-G_{i}\right)^{2}+\left(B-B_{i}\right)^{2}+K^{2}\left(N_{j-1}-N_{j}\right)^{2}}
$$

donde $R, G, B$ son los niveles de intensidad de la imagen analizada; $R_{i}, G_{i}, B_{i}$ corresponden a los valores almacenados en la tabla de búsqueda; la expresión (10) toma también en cuenta la continuidad del orden de franja pues $K$ es un parámetro de regularización para tomar en cuenta la variación continua de la distribución de esfuerzos y en este caso se utilizó un valor de $K=75 ; N_{j-1}$ es el valor del orden de franja del pixel vecino tomando en cuenta si el escaneo se realiza vertical u horizontalmente y $N_{j}$, cualquier valor posible en la tabla de búsqueda.

\subsection{Elección de semilla}

Con la consideración de tomar en cuenta los valores de orden de franja previamente asignados, resulta importante la elección del primer valor que será el punto de partida para el escaneo de toda el área de interés de la imagen. Dicho valor se conoce como semilla. Evidentemente, al ser el valor que será referencia para asignar a los pixeles vecinos (que a su vez serán referencia para otros pixeles y así sucesivamente), no posee un referente para su elección. De manera que hay que tener en mente dicha asignación, por lo que se han propuesto varios esquemas de escaneo $[30,31,32]$.

En este trabajo, las imágenes se escanearon de la siguiente manera: ya que las imágenes presentan un arreglo matricial, se realiza una asignación del orden de franja sin tomar en cuenta su continuidad para elegir un pixel como valor semilla. Para su elección se selecciona el pixel que más cercano haya estado a la curva de la tabla de calibración. Con dicho valor asignado se escanea la columna a la que pertenece respetando la continuidad y finalmente se barre el resto de la imagen hacia la derecha e izquierda.

\subsection{Generación de imágenes fotoelásticas artificiales}

Para la programación de la técnica y la validación de resultados es necesario contar con imágenes representativas del fenómeno de la fotoelasticidad que sirvan como datos de entrada. Se optó por generar imágenes artificiales, ya que permiten conocer la distribución de esfuerzos dentro de la geometría del material; esto permite validar el software al conocer con antelación dicha distribución, y ofrecen la posibilidad de probar con distintas geometrías y cargas.

A través de una simulación de elemento finito con el software COMSOL Multiphysics, se obtuvo la distribución de la diferencia de los esfuerzos principales en algunos modelos de 
geometría simple. En particular: un disco en compresión uniaxial, y una probeta cuadrada con un orificio central. A partir de los datos arrojados, se calculó la diferencia de esfuerzos principales en cada nodo del modelo. Con dicha información se obtuvo la intensidad de la luz transmitida (ecuación (9)) por el polariscopio circular en su configuración de campo oscuro, tal como la captaría una cámara digital que lo separa en los canales $R, G, B$, asumiendo que la fuente de luz blanca está formada por tres longitudes de onda de la misma intensidad, correspondientes a los colores rojo, verde y azul (similar a la de una fuente de luz fluorescente) con las longitudes de onda mencionadas en el apartado 2.2, y que no hay dispersión de la birrefringencia, por lo que se asume que el material en cuestión presenta un coeficiente fotoelástico constante.

Los datos se importaron hacia MATLAB para, mediante una interpolación, obtener el valor de los pixeles para los canales $R, G, B$, y así obtener la imagen fotoelástica artificial. En la figura 4 se muestra una representación de la geometría simulada (4a) y la imagen fotoelástica artificial generada con los datos de la probeta en forma de disco de polimetilmetacrilato de $5 \mathrm{~cm}$ de radio y $0.5 \mathrm{~cm}$ de espesor, sometida a una carga uniaxial de $200 \mathrm{~N}$ (4b).
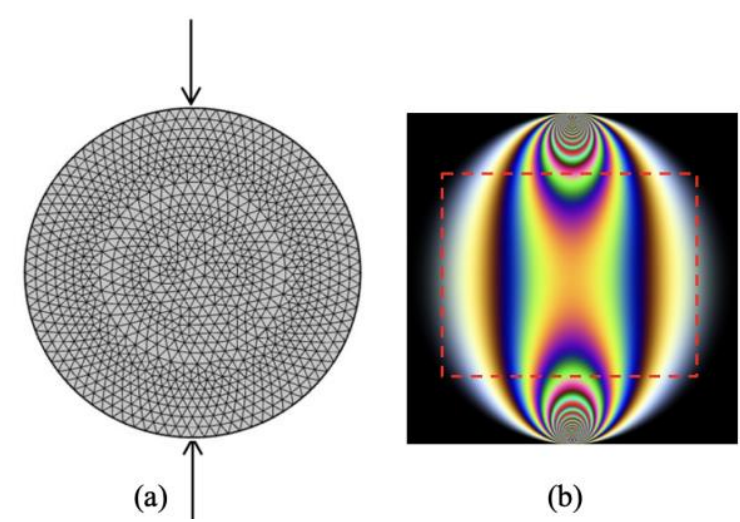

(b)

Figura 4. Representación del modelo seleccionado para la simulación (a). Imagen fotoelástica artificial de un disco en compresión (b) (se muestra enmarcada en un recuadro cierta área de interés seleccionada para el escaneo).

Siguiendo este mismo proceso se calculó otra imagen de geometría sencilla considerando el mismo material y espesor: probeta cuadrada de 5 $\mathrm{cm}$ de lado con un orificio en el centro de $5 \mathrm{~mm}$ de radio (figura 5a) sometido a compresión con una carga de $250 \mathrm{~N}$ (véase la figura $5 \mathrm{~b}$ ).

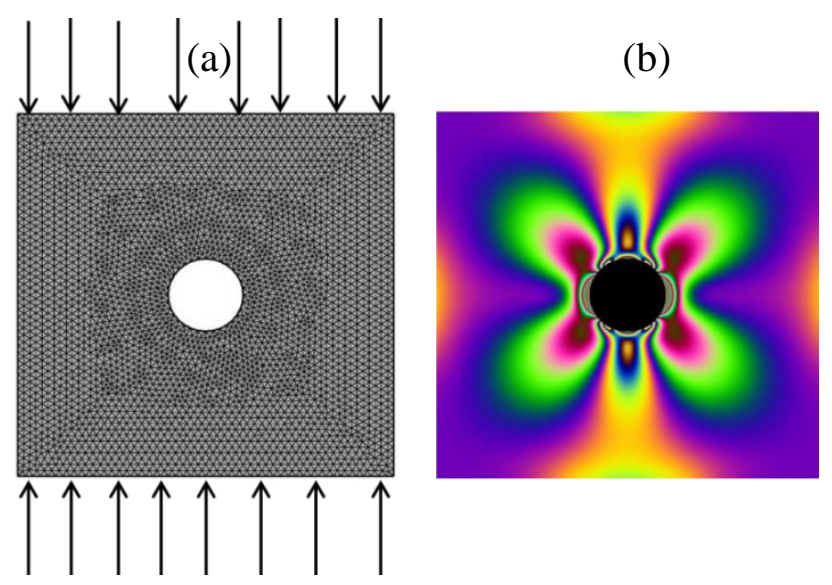

Figura 5. (a) Representación del modelo seleccionado para la simulación del cuadro a compresión, (b) Imagen fotoelástica artificial de una probeta cuadrada en compresión de $5 \mathrm{~cm}$ de lado sometido a una carga de $250 \mathrm{~N}$. 

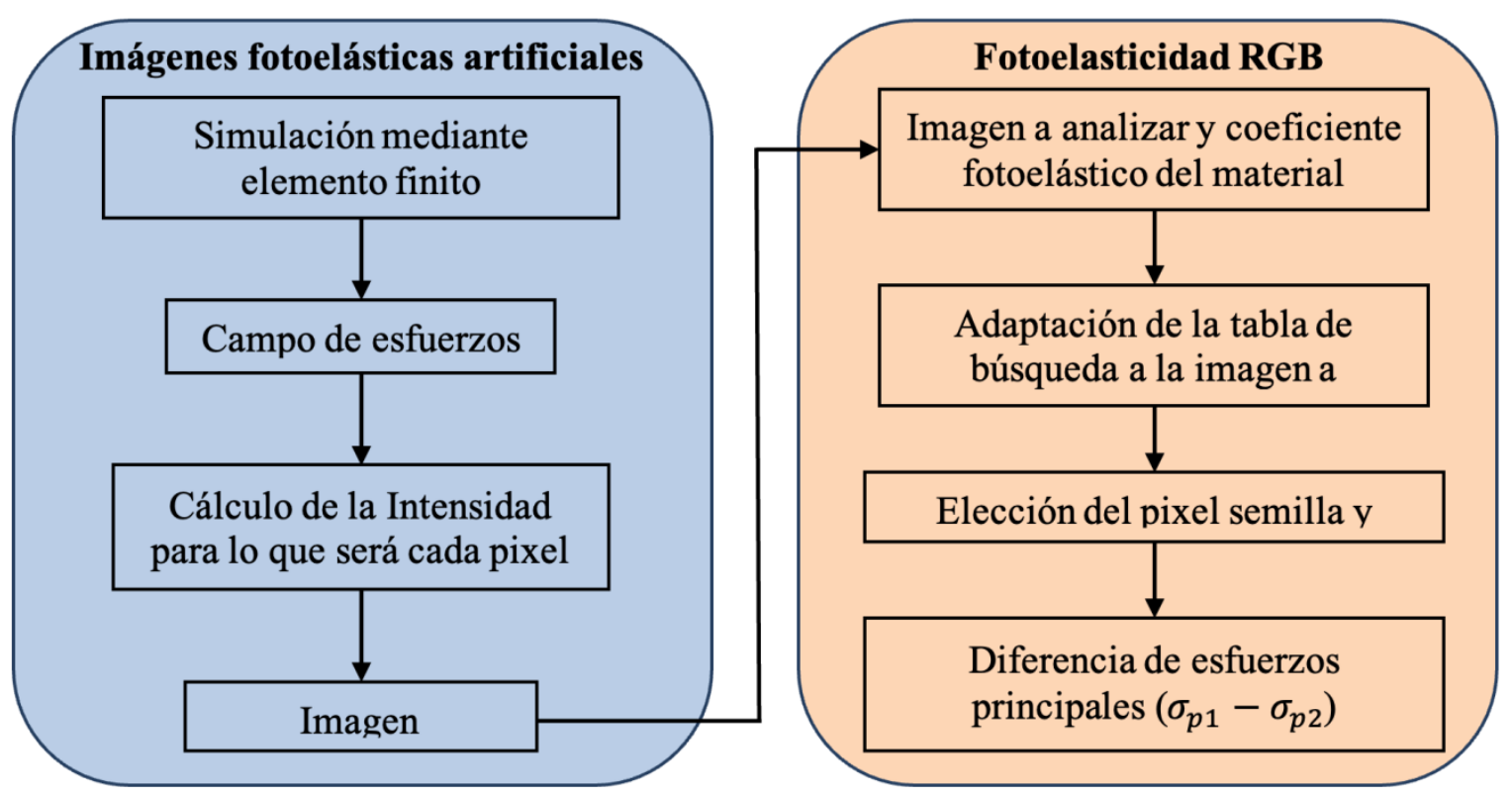

Figura 6. Esquema de la metodología utilizada.

En la figura 6 se puede observar un esquema de la metodología empleada para este trabajo.

\section{Resultados}

Las imágenes fotoelásticas, generadas artificialmente, sirven de dato para el código desarrollado en MATLAB. Este permite seleccionar un área de interés dentro de la imagen a analizar, y obtener información mediante el uso de la ecuación (2). Con la información del número de franja asociado a cada pixel de la imagen considerada se calculó la diferencia de esfuerzos principales mediante la relación (1).
En la figura 5b (imagen fotoelástica artificial de un disco de polimetilmetacrilato a compresión) se muestra enmarcada en un recuadro cierta área de interés seleccionada para el análisis. Al comparar la intensidad de los canales R, G, B de cada pixel del área seleccionada con los de la tabla de búsqueda según lo expuesto en el apartado 2.3 y minimizando el valor de la función (10) se asigna a cada pixel evaluado un valor de orden de franja. Con esto se obtiene también la diferencia de esfuerzos principales en cada punto de la imagen analizada. Se presenta la distribución de esfuerzos calculada, en pascales, en dicha sección de la imagen fotoelástica artificial del disco a compresión en la figura 7. 


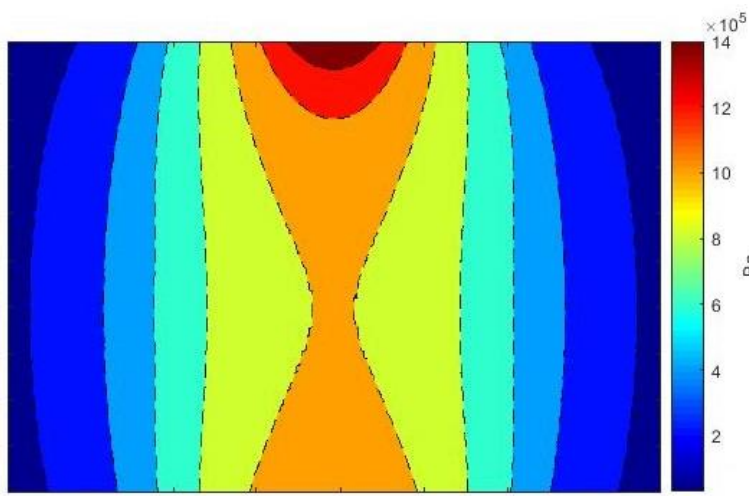

(a)

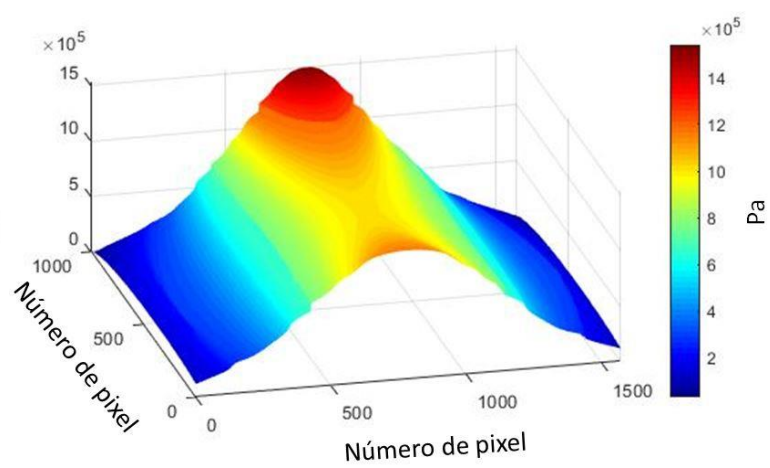

(b)

Figura 7. Diferencia de esfuerzos principales en la sección del disco analizada (en Pa). Presentada en un gráfico de curva de nivel (a), y de superficie tridimensional (b).

El gráfico señala los valores calculados en curvas de nivel (figura 7a) y en la figura 8 b, la misma distribución en un gráfico de superficie donde la altura está en relación con los valores obtenidos. Se puede observar que dicha distribución varía de forma continua a lo largo de la imagen analizada.

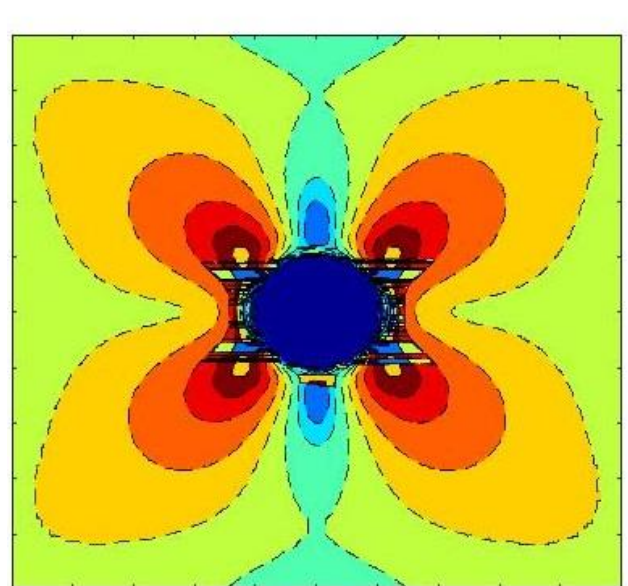

(a)
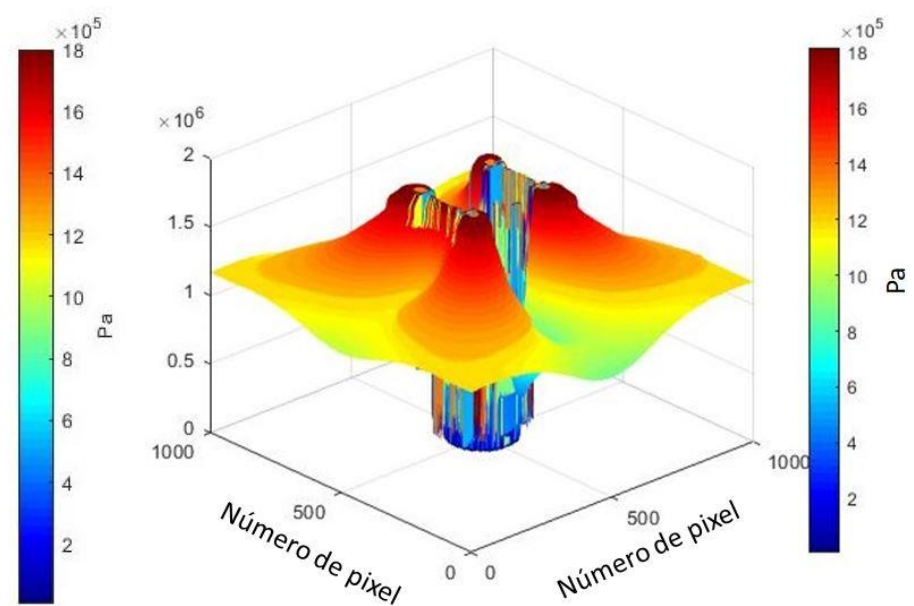

(b)

Figura 8. Diferencia de esfuerzos principales en la probeta del cuadro a compresión (en Pa). Se presenta en un gráfico de curva de nivel (a), y de superficie tridimensional (b).

Se hizo una comparación de los resultados obtenidos con los de la simulación en COMSOL Multiphysics de la siguiente manera: después de tener asignado un valor de la diferencia de esfuerzos principales en cada pixel de la imagen analizada, se interpolaron los datos para compararlos con puntos específicos de la simulación por elemento finito. Se obtuvo, para cada punto comparado, un error relativo según la ecuación (11), donde el esfuerzo promedio es precisamente un promedio de la diferencia de esfuerzos principales en el modelo simulado. Para el análisis de la imagen mencionada, el 94\% de los puntos comparados presentan un error relativo menor al $5 \%$; además, un $5 \%$ de puntos tienen un error relativo entre 5 y $10 \%$; en 


\section{Revista de Ciencias Tecnológicas (RECIT): Volumen 4 (4): 399-411}

promedio, el error relativo en toda la imagen es de un $2 \%$.

$$
\operatorname{er}(\%)=\frac{\text { dato escaneo }- \text { dato simulación }}{\text { esfuerzo promedio }}(100)
$$

También se presenta en la figura 8 el resultado de la asignación de la diferencia de esfuerzos principales en la imagen artificial de la probeta de polimetilmetacrilato cuadrada con un orificio central. Se muestra la distribución en curvas de nivel (figura 8a), mientras que la figura $8 \mathrm{~b}$ esquematiza la misma distribución en una gráfica de superficie tridimensional. Se puede observar que dicha distribución varía de forma continua a lo largo de la imagen analizada tal como se pretendía; también se presentan ciertas discontinuidades donde existen concentraciones de esfuerzo, así como en una zona sin material en el centro del espécimen. Para los resultados de esta imagen, el promedio del error relativo fue $3.5 \%$, el porcentaje de los puntos comparados con un error relativo menor a $5 \%$ fue $97 \%$ y también un $2.4 \%$ de puntos tienen un error mayor a $20 \%$.

\section{Discusión y conclusiones}

Se desarrolló un algoritmo en MATLAB que calcula la distribución de la diferencia de esfuerzos principales a través de una imagen fotoelástica, mediante el método de la fotoelasticidad RGB. El programa puede presentar los resultados en gráficos estilo mapa de colores, así como la facilidad de evaluar algún punto de interés. También, se generaron satisfactoriamente imágenes artificiales que muestran el fenómeno de la fotoelasticidad con la ayuda de los datos arrojados por la simulación de elemento finito de probetas de geometría sencilla. Además, fue posible crear una tabla de búsqueda de manera teórica y adaptarla a las imágenes requeridas según requiere la técnica implementada. La adaptación de la tabla de búsqueda arrojó buenos resultados en la asignación del retardo relativo. Finalmente, la comparación de la distribución de la diferencia de esfuerzos principales arrojada por el algoritmo al escanear las imágenes, con los datos de la simulación de elemento finito dio un buen grado de confiabilidad en las imágenes utilizadas. Es importante señalar que el código al que se hace referencia se encuentra disponible en un trabajo más extenso [33].

Hay que mencionar rango de la tabla de búsqueda es importante ya que es el rango en el que podemos comparar y asignar un número de franja a cada pixel y, por lo tanto, un valor de la diferencia de esfuerzos principales. El método utilizado hace una comparación de las intensidades en los canales R, G, y B entre la tabla y la imagen; en otras palabras, lo que hace es encontrar el color más parecido aún si algún valor de orden de franja de la imagen fotoelástica excede la tabla de búsqueda utilizada. A esto se le atribuyen ciertos valores del error relativo calculado en las imágenes.

Sin embargo, es importante mencionar que el orden de franja máximo detectable en el espécimen está íntimamente relacionado con la fuente de luz empleada. De manera general, en los métodos fotoelásticos, se han utilizado lámparas incandescentes que presentan un espectro de emisión continuo; esto genera limitaciones en cuanto al orden de franja máximo detectable debido a que la señal RGB decrece rápidamente mientras incrementa el orden de franja. Para este tipo de fuente de luz, se utiliza, por lo general, un orden de franja máximo igual a tres. Con otro tipo de fuente, como luz formada por solo con tres longitudes de onda o mediante filtros para los canales RGB se puede aumentar el orden de franja máximo. Una opción más sencilla es una lámpara fluorescente de espectro discreto donde el orden máximo de franja puede llegar hasta doce $[19,24]$. Se puede elaborar la tabla de búsqueda con un mayor orden de franja y así trabajar con ese tipo de fuentes de luz. Aun así, se menciona que hay mejores resultados para órdenes de franja bajos $[26,34]$ a menos que se utilice una tabla experimental. 
Otra situación de interés se da en zonas de alta concentración de esfuerzos y en las de baja diferencia de esfuerzos principales. Por lo general donde la concentración de esfuerzos es elevada, la asignación del orden de franja dependerá en gran medida de la resolución y el tamaño de pixel del equipo de adquisición ya que se deben analizar una gran cantidad de franjas y se requiere también una gran cantidad de datos que ofrecen los pixeles de la imagen. De igual manera, se presenta otra situación en las zonas donde la diferencia de esfuerzos principales es baja. Esto parece deberse a que, en la región de órdenes de franja bajos de la tabla de búsqueda, las intensidades de los tres canales R, G, y B generan tonos similares que pueden influir en los resultados.

Por todo lo anterior, implementar una tabla de calibración experimental puede ser de gran utilidad para extender las capacidades del software así como dotarlo de métodos de escaneo y reducción de ruido para geometrías más complejas [31, 32].

Finalmente, es necesaria la validación el software mediante imágenes experimentales y así facilitar la ejecución de nuevas investigaciones. Es importante también trabajar en la separación de la diferencia de esfuerzos principales, para acceder al tensor de esfuerzos y a través de la ecuación de comportamiento obtener información de las deformaciones en la probeta. También es posible ampliar la técnica, y utilizarla no solo en probetas de materiales fotoelásticos sino en otro tipo de materiales mediante algún recubrimiento que sí tenga dicha propiedad.

\section{Agradecimientos}

Los autores agradecen de manera especial al Centro de Investigación en Materiales Avanzados y al laboratorio de Prototipos e integridad mecánica del Departamento de Metalurgia e Integridad Estructural. Así como al programa de maestría en ciencia de materiales y al programa de becas de posgrado del CONACYT.

\section{Reconocimiento de autoria}

Rubén Castañeda Balderas: Conceptualización; Metodología; Supervisión. Alberto Díaz Díaz: Supervisión; Recursos; Revisión. David Trejo Carrillo: Conceptualización; Software; Borrador original; Escritura.

\section{Referencias}

[1] W. N. Sharpe, Springer handbook of experimental solid mechanics, New York: Springer Science \& Business Media, 2008. https://doi.org/10.1007/978-0-387-30877-7

[2] D. Post, «Photoelasticity» Experimental Mechanics, vol. 19, no 5, pp. 176-192, 1979. https://doi.org/10.1007/BF02324263

[3] J. F. Doyle, Modern experimental stress analysis: completing the solution of partially specified problems, John Wiley \& Sons, 2004. https://doi.org/10.1002/0470861584

[4] E. A. Garrido, Estudio de esfuerzos mecánicos en materiales oftálmicos mediante fotoelasticidad. Tesis de maestría, UNiversidad Autónoma Metropolitana de Azcapotzalco. 2017. http://hdl.handle.net/11191/5752.

[5] K. Ramesh y V. Ramakrishnan, «Digital photoelasticity of glass: A comprehensive review» Optics and Lasers in Engineering, vol. 87, pp. 59-74, 2016. https://doi.org/10.1016/j.optlaseng.2016.03.017

[6] M. Dundovic, K. Morkovic y M. V. Z. Franulovic, «Digital light processing in photoelastic models production for material behavior modeling» Procedia Structural integrity, vol. 31, pp. 111-115, 2021. https://doi.org/10.1016/j.prostr.2021.03.018 


\section{Revista de Ciencias Tecnológicas (RECIT): Volumen 4 (4): 399-411}

[7] J. Urango, G. Carmen, J. Briñez y A. Restrepo, «Validación del uso de la fotoelasticidad como herramienta para los cursos de mecánica de sólidos» EIA, vol. 14, nº 28, pp. $117 \quad$ - $131, \quad 2017$. https://doi.org/10.24050/reia.v14i28.1145

[8] A. Rey, Modelado del comportamiento mecánico no lineal de un policarbonato en el estado vítreo. Tesis de doctorado, Centro de Investigación en Materiales Avanzados, S. C., Chihuahua, Chih. ,2019.

[9] Z. Ren, H. Xie y Y. Ju, «Determination of stress and strain fields in porous structures by photoelasticity and digital image correlation techniques» Polymer Testing, $\mathrm{n}^{\mathbf{o}}$ 102, p. 107315, 2021.

https://doi.org/10.1016/j.polymertesting.2021.10 $\underline{7315}$

[10] P. C. Sung, W. C. Wang y C. H. L. G. T. Hwang, «A low-level stress measurement method by integrating white ligth photoelasticity and spectrometry» Optics \& Laser Technology, vol. 98, pp. 33-45, 2018. https://doi.org/10.1016/j.optlastec.2017.07.022

[11] E. Patterson, «Automated photoelastic analysis» Strain, vol. 1, no 24, pp. 15-20, 1988. https://doi.org/10.1111/j.1475-

1305.1988.tb00650.x

[12] C. A. Magallañes, «Computational methods of phase shifting to stress measurement with photoelasticity using plane polariscope» Optik, vol. 130, pp. 213-226, 2017. https://doi.org/10.1016/j.ijleo.2016.11.037

[13] J. C. R. A. Briñez y F. López, «Estudios de fotoelasticidaad:desarrollos y aplicaciones,» Revista politécnica, $\mathrm{n}^{\circ}$ 16, pp. 27-36, 2013. https://doi.org/10.33571/rpolitec

[14] K. Ramesh y S. Sasikumar, «Digital photoelasticity: Recent developments and diverse applications» Optics and Lasers in Engineering, vol. 135, pp. 106-186, 2020. https://doi.org/10.1016/j.optlaseng.2020.106186

[15] M. Ramji y R. Prasath, «Sensivity of isoclinic data using various phase shifting techniques in digital photoelasticity towards generalized error sources» Optics and Lasers in Engineering, $\mathrm{n}^{\mathrm{o}}$ 49, pp. 1153-1167, 2011. https://doi.org/10.1016/j.optlaseng.2011.05.007

[16] S. Yoneyama, M. Shimizu, J. Gotoh y M. Takashi, «Photoelastic Analysis with a Single Tricolor Image» Optics and Lasers in Engineering, $\mathrm{n}^{\mathrm{o}}$ 29, pp. 423-435, 1998. https://doi.org/10.1016/S0143-8166(97)00107-3

[17] A. Ajovalasit, G. Petrucci y M. Scafidi, «Review of RGB photoelasticity» Optics and Lasers in Engineering, $\mathrm{n}^{\circ}$ 68, p. 58.73, 2015. https://doi.org/10.1016/j.optlaseng.2014.12.008

[18] J. Briñez de León, H. Fandiño, A. Restrepo y J. Branch, «Análisis de resolución en imágenes de fotoelasticidad: caso carga dinámica,» VISIÓN ELECTRÓNICA, vol. 11, nº 1, 2017. https://doi.org/10.14483/22484728.12789

[19] D. Swain, J. Philip y A. Pillai, «A modified regularized scheme for isochromatic demodulation in RGB photoelasticity» Optics and Lasers in Engineering, vol. 61, pp. 39-51, 2014.

https://doi.org/10.1016/j.optlaseng.2014.04.009

[20] J. A. Quiroga y G.-P. J. A. García-Botella, «Improved method for isochromatic demodulation by RGB calibration» Applied optics, vol. 41, $\mathrm{n}^{\mathrm{o}}$ 17, pp. 3461-3468, 2002. https://doi.org/10.1364/AO.41.003461

[21] B. N. Simon, T. Kasimayan y K. Ramesh, «The influence of ambient illumination on colour adaptation in three fringe photoelasticity» Optics and Lasers in Engineering, vol. 49, n ${ }^{\circ}$ 2, pp. 258- 


\section{Revista de Ciencias Tecnológicas (RECIT): Volumen 4 (4): 399-411}

264,

2011. https://doi.org/10.1016/j.optlaseng.2010.09.018

[22] A. Ajovalasit, S. Barone y G. Petrucci, «Towards RGB photoelasticity: full-field automated photoelasticity in white light» Experimental Mechanics, vol. 35, no 3, pp. 193200, 1995. https://doi.org/10.1111/j.14751305.2008.00571.x

[23] D. Swain, B. Thomas, J. Philip y A. Pillai, «Novel calibration and color adaptation schemes in three-fringe RGB photoelasticity» Optics and Lasers in Engineering, $n^{\circ}$ 66, pp. 320-329, 2015. https://doi.org/10.1016/j.optlaseng.2014.10.007

[24] A. Ajovalasit, G. Petrucci y M. Scafidi, «RB photoelasticity: review and improvements» Strain, no 46, pp. 137-147, 2010. https://doi.org/10.1111/j.1475-

$\underline{1305.2008 .00571 . x}$

[25] B. N. Simon, T. Kasimayan y K. Ramesh, «The influence of ambient illumination on colour adaptation in three fringe photoelasticity» Optics and lasers in engineering, vol. $49, \mathrm{n}^{\mathrm{o}} 2$, pp. 258264,

2011. https://doi.org/10.1016/j.optlaseng.2010.09.018

[26] K. Ramesh y A. Pandey, «An improved normalization technique for white light photoelasticity» Optics and Lasers in Engineering, vol. 109, pp. 7-16, 2018. https://doi.org/10.1016/j.optlaseng.2018.05.004

[27] P. M. Ch, V. Ramakrishnan y R. Krishnamurthi, «Assessment of fringe pattern normalisation for twelve fringe pgotoelasticity,» Advancement of Optical Methods in Experimental Mechanics, vol. 3, pp. 295-299, 2017. https://doi.org/10.1007/978-3-319-416007_37

[28] M. M. D. P. Sudharshan Duth, «Color detection in RGB modeled images using
MATLAB» International Journal of Engineering \& Technology, vol. 7, pp. 29-33, 2018. https://doi.org/10.14419/ijet.v7i2.31.13391

[29] The MathWorks, Inc., «Understanding Color Spaces and Color Space Conversion» [En línea].

Available: https://www.mathworks.com/help/images/under standing-color-spaces-and-color-spaceconversion.html. [Último acceso: Octubre 2021].

[30] V. Ramakrishnan y K. Ramesh, «Scanning schemes in white light photoelasticity - part $\mathrm{i}$ : Critical assessment of existing schemes» Optics and Lasers in Engineering, vol. 92, pp. 129-140, 2017.

https://doi.org/10.1016/j.optlaseng.2016.06.016

[31] K. Ramesh, V. Ramakrishnan y R. C., «New initiatives in single-colour image-based fringe order estimation in digital photoelasticity» Journal of Strain Analysis, pp. 1-17, 2015. https://doi.org/10.1177/0309324715600044

[32] V. Ramakrishnan y K. Ramesh, «Scanning schemes in white light photoelasticity - Part II: Novel fringe resolution guided scanning scheme» Optics and Lasers in Engineering, 2016. https://doi.org/10.1016/j.optlaseng.2016.05.010

[33] D. Trejo, Software de fotoelasticidad RGB en placas de materiales birrefringentes al esfuerzo, Tesis de maestría, Centro de Investigación en Materiales Avanzados, S. C., Chihuahua, Chih. ,2021.

[34] K. Ramesh y A. Pandey, «Development of a New Normalization Technique for Twelve Fringe Photoelasticity (TFP),» Advancement of Optical Methods \& Digital Image Correlation in Experimental Mechanics, vol. 3, pp. 177-180, 2018. https://doi.org/10.1007/978-3-319-97481$\underline{1 \_23}$ 


\section{Revista de Ciencias Tecnológicas (RECIT): Volumen 4 (4): 399-411}

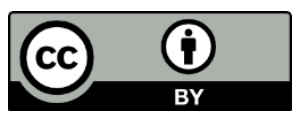

Este texto está protegido por una licencia Creative Commons 4.0

Usted es libre para Compartir - copiar y redistribuir el material en cualquier medio o formato - y Adaptar el documento - remezclar, transformar y crear a partir del material — para cualquier propósito, incluso para fines comerciales, siempre que cumpla la condición de:

Atribución: Usted debe dar crédito a la obra original de manera adecuada, proporcionar un enlace a la licencia, e indicar si se han realizado cambios. Puede hacerlo en cualquier forma razonable, pero no de forma tal que sugiera que tiene el apoyo del licenciante o lo recibe por el uso que hace de la obra.

$\underline{\text { Resumen de licencia - Texto completo de la licencia }}$ 